\title{
Pensar y actuar metodológicamente: Una expe- riencia de investigación mediante un estudio de casos cualitativo
}

\author{
Albina CUADRADO FERNÁNDEZ ${ }^{1}$ \\ Universidad Complutense de Madrid \\ alcuadra@edu.ucm.es
}

Recibido: $30 / 06 / 13$

Aceptado: 06/11/13

\section{RESUMEN}

En este artículo se presenta una experiencia práctica en relación con el proceso de investigación de una tesis doctoral en educación musical con el propósito de ofrecer un marco de referencia para futuras investigaciones sobre la música u otras artes en la escuela. Para ello, se realiza una revisión de los fundamentos metodológicos del trabajo y se analiza el diseño de la investigación.

Palabras clave: Metodología cualitativa, estudio de casos, diseño de la investigación.

\section{Referencia normalizada}

CUADRADO FERNÁNDEZ; A. (2013). "Pensar y actuar metodológicamente: Una experiencia de investigación mediante un estudio de casos cualitativo". En Arteterapia: Papeles de arteterapia y educación artística para la inclusión social Vol.: 8. Páginas 191-210.

\section{SUMARIO}

Introducción. Paradigma interpretativo y metodología cualitativa. El estudio de casos. Las técnicas de recogida de datos. El análisis y la interpretación de los datos. El proceso de la investigación: pensar y actuar metodológicamente. Referencias bibliográficas.

\section{Thinking and acting methodologically: A research experience of qualita-} tive case study

\begin{abstract}
This article presents a practical experience as part of the research process of a doctoral thesis in music education with the aim of contributing to provide with a framework for future researches about music or other arts in the school. For it, a review of the methodological foundations of this work is included along with an analysis of the design of this investigation.
\end{abstract}

Keywords: Qualitative methodology, case study, design of research.

\footnotetext{
${ }^{1}$ Profesora en la Universidad Complutense de Madrid. Cantante profesional especialista en música medieval.
} 


\section{CONTENTS}

Introduction. Interpretive paradigm and qualitative methodology. Case study. Data collection methods. Data analysis and interpretation. Research process: thinking and acting methodologically. References.

\section{INTRODUCCIÓN}

Cuando se comienza una investigación, el solo hecho de tener que enfrentarse a la metodología puede resultar intimidatorio. Tal era mi percepción en los prolegómenos del trabajo que ha servido de sustento para este artículo ${ }^{2}$. A medida que me adentraba en la investigación fui encontrando en la metodología un referente y un aliado. Aún hoy conservo vívida la impresión que produjo en mí un primer acercamiento a la obra de Elliot Eisner, Liora Bresler o Saville Kushner, entre otros autores. Las lecturas y relecturas de sus trabajos me iban revelando una metodología a mi alcance que servía para buscar respuestas a los interrogantes en torno al tema de estudio, y que preveía y contemplaba las contingencias de las interacciones con las personas que participaban en la investigación. Una metodología que, lejos de llevar hacia una metodolatría ciega, daba implícitamente la opción de poder pensar metodológicamente, pero también creativamente con y desde ella, y me daba la posibilidad de situarme así en el camino de poder encontrar mi propia voz como investigadora a medida que discurría el proceso de la investigación.

La metodología es la herramienta del investigador. La elección de la herramienta no es algo trivial ni anecdótico puesto que, en las características de la herramienta y en el uso que se haga de ella puede verse comprometida la respuesta a las demandas de la investigación en función de su naturaleza. El símil del cocinero en su cocina puede servir de ejemplo. Podemos contar con unos ingredientes de primera calidad y con unas cacerolas confeccionadas con unos materiales de última generación y en perfecto estado de conservación. Podemos, además, estar en posesión de una receta contrastada y mejorada durante generaciones, pero si variamos el tiempo de cocción o la temperatura, si incrementamos o disminuimos la sal, el aceite, o las hierbas aromáticas, podremos llevar, o no, el plato a la mesa en las mejores condiciones.

Conocer la metodología proporciona al investigador la posibilidad de manejarla adecuadamente y de ser fructíferamente creativo al respecto.

\footnotetext{
2 "El canto en las aulas de música de educación primaria: un análisis interpretativo de diversas prácticas docentes". Tesis doctoral defendida el día 8 de abril de 2013 en la Sala de Grados de la Facultad de Educación de la Universidad Complutense de Madrid (UCM).
} 


\section{PARADIGMA INTERPRETATIVO Y METODOLOGÍA CUALITATIVA}

La metodología responde a una cuestión esencial a la que ha de dar respuesta el investigador a la hora de abordar su trabajo y no ha de ser contemplada aisladamente, ya que toda investigación se enmarca dentro de un enfoque y de una manera de mirar que se establece en función de una estructura de pensamiento denominada "paradigma". El paradigma no es una teoría, es más bien una manera de concebir la realidad -la realidad social, en esta investigación- que cuando es aplicada puede conducir al desarrollo de la teoría. Un paradigma científico es un sistema de creencias básicas a las que se adhiere el investigador, según Guba y Lincoln (1994:108) reelaborando el concepto creado por Kuhn (1962/1971), acerca de cuestiones ontológicas (la forma y naturaleza de la realidad y qué puede saberse sobre la misma), epistemológicas (la relación entre el investigador y aquello que cree que puede ser sabido) y metodológicas (la manera en que se va a intentar averiguar aquello que se cree que puede saberse). Así pues, la conceptualización de paradigma no se limita solamente a esas cuestiones metodológicas, sino que guía al investigador, además, en aspectos ontológicos y epistemológicos fundamentales (Guba \& Lincoln, 1994:105). Esas tres cuestiones están relacionadas de manera que el supuesto ontológico que asume el investigador debería llevar implícitas posturas en concordancia, tanto desde el punto de vista epistemológico como metodológico.

La corriente de pensamiento que da forma al paradigma interpretativo hunde sus raíces en el movimiento idealista que surge de la filosofía de Kant, cuyo pensamiento dirige la atención a la subjetividad humana, a sus posibilidades y limitaciones cognoscitivas e introduce la figura del observador o, lo que es lo mismo, la figura del sujeto humano como sujeto de conocimiento. Para Kant (1781/1998), nuestra representación de las cosas, tal como nos son dadas, no se establece como si fueran cosas en sí mismas, sino como fenómenos que se regulan por nuestra manera de representarlos. Desde este punto de vista, como escribe Bresler (2006a), toda experiencia estaría mediada por la mente, toda inteligencia estaría imbuida de ella y limitada a la interpretación y a la representación, y todo lo que podríamos llegar a conocer serían fenómenos.

A comienzos del siglo XX surge una corriente de pensamiento en el marco de la antropología que entiende la realidad como algo múltiple, cambiante, impredecible e interpretable. Así, el aula no sería un universo fijo, ni estable, ni unidimensional. Sería un universo lleno de paradojas y contradicciones, tanto en el espacio, de un aula a otra, como en el tiempo, de un momento a otro, de un día a otro. Según este nuevo concepto de realidad en las ciencias sociales, ésta habría de ser reconstruida desde la interpretación. Esta idea de realidad tiene que ver con un concepto de ciencia social más humilde, que no busca leyes invariables, eternas y universales. Para Eisner (1998) no sería una ciencia omnisciente, sino democrática y ecuménica, una ciencia que aborda la realidad de abajo hacia arriba, una ciencia interpretativa en busca de significado, de construcciones. Se 
trata de una ciencia que asume la interacción entre el investigador y lo investigado de manera que ya no se contempla el objeto y el sujeto como realidades absolutas, sino que aparecen como dos emergencias inseparables, no duales, con una relación compleja de la que emerge la idea del carácter incompleto de cada una.

Esta revolución paradigmática, en definitiva, supone un cambio radical, no solamente porque se renueva la concepción del objeto, sino porque revierte en las perspectivas epistemológicas del sujeto observador científico dando lugar a una nueva epistemología que reclama el lugar tanto de la incertidumbre como de la dialógica y demanda y aporta los medios para la autocrítica y para la revisión permanente, a la vez que concibe, como ha quedado expuesto, un nuevo concepto de ciencia social.

Desde estos presupuestos, la figura del investigador participa del mismo concepto de humildad que se le atribuye a la ciencia. Como escribe Eisner (1998), el investigador es más cauto, ya que es consciente de que lo que se llega a saber está influido por las herramientas que se poseen. Además de asumir una interacción con lo investigado, el investigador sabe que él mismo es el instrumento principal de la investigación. No trata, en ningún modo, de examinar conductas, sino de percibir su presencia e interpretar su significado. A la vez, desarrolla la capacidad de compartir lo que ha experimentado con quienes no estaban allí por medio de un lenguaje expresivo en el que tienen cabida también los sentimientos y las emociones, que no son considerados como enemigos de la cognición, sino todo lo contrario. El investigador sabe que la necesidad de una pretendida objetividad puede conducir al camuflaje, por eso no evita lo personal, lo literario o lo poético, sino que los considera fuente de saber, y pone conscientemente su propia firma en el trabajo que realiza. El esfuerzo del investigador no va dirigido a buscar la uniformidad ni la estandarización, sino que se dirige a dotar de sentido a sus acciones dependiendo de la naturaleza del problema por el que está interesado, del talento que posea y del contexto en el que trabaje. Además, puesto que las acciones no son predecibles, ese esfuerzo se dirigirá a hacer suyos los conceptos de flexibilidad, ajuste e interacción que son, según Eisner (1998), las señas de identidad de la metodología cualitativa porque no hay reglas ni rutinas que él pueda seguir, sino que hay deseos, propósitos y la necesidad de conectar con lo que es importante.

Kushner (2002) coincide con estos planteamientos en relación con la idea de que es necesario que el investigador encuentre su propia voz en la investigación, para lo que sugiere no practicar únicamente metodología sino pensar independientemente de ella sin que esto vaya en detrimento de la necesidad del investigador de ser metodológico.

La metodología asociada al paradigma interpretativo es la metodología cualitativa, cuyos métodos son conocidos como exploratorios, descriptivos o interpretativos.

Esta metodología tiene un enfoque inductivo. No busca leyes naturales eternas, lo que no quiere decir que a la investigación cualitativa no le interesen factores universales, sino que toma otro camino para descubrirlos. No busca factores universales abstractos, sino factores universales "concretos" a los que se llega 
estudiando un caso específico en detalles, comparándolo con otros que han sido igualmente estudiados. No pretende generalizar, ya que la generalización implica relaciones causa-efecto y la metodología cualitativa no intenta establecer relaciones causales sino que se centra en lo teleológico ${ }^{3}$, lo intencional. La investigación cualitativa no es unidireccional ni estática y deja siempre espacio para el debate y la diferencia. Tiene un ritmo no siempre predecible, aunque no por ello haga apología de planteamientos ilusionados o descuidados. Los sellos de la metodología cualitativa son, para Eisner (1998), la flexibilidad, el ajuste y la interacción, con una orientación fenomenológica.

La metodología cualitativa es emergente en cuanto que responde a la naturaleza del campo de la investigación. La combinación de diferentes métodos, perspectivas o materiales empíricos, se entiende como una estrategia que añade rigor, amplitud y profundidad al trabajo del investigador, de ahí que se pueda concebir la investigación cualitativa como un bricolaje y al investigador como un bricoleur, metáfora propuesta por algunos investigadores como Denzing y Lincoln (1994). Así pues, la investigación cualitativa permite numerosos enfoques cuyas diferencias fundamentales vienen marcadas por las opciones que adopte el investigador desde el punto de vista ontológico, epistemológico y metodológico. Desde finales de la década de 1970 y principios de la de 1980, la investigación cualitativa ha quedado establecida como un medio idóneo, y cada vez más relevante, para abordar la investigación en educación, puesto que ésta resulta influenciada por valores socioculturales.

La adecuación de la investigación cualitativa para la investigación en educación musical se fundamenta, según Aróstegui (2004:7-8), en tres principios ${ }^{4}$ : en primer lugar en el "relativismo" ya que el desarrollo de los problemas estéticos en educación musical no debería buscar "respuestas correctas" ni explicar los fenómenos, pero sí interpretarlos, ya que eso es lo que constituye uno de los sellos de identidad de la investigación cualitativa, y además, la educación musical y la investigación cualitativa suponen objetividad y subjetividad al mismo tiempo, lo que no significa arbitrariedad, sino el reconocimiento de realidades múltiples; en segundo lugar se basa en el "enlace entre teoría y práctica" ya que la búsqueda para entender el contexto social establece una dirección "bidireccional" entre la práctica en educación musical y la investigación. La investigación cualitativa persigue tanto la descripción como la interpretación de la práctica con la esperanza de transformar dicha práctica para que la investigación y la práctica se acerquen; en tercer lugar se fundamenta en la "democracia", ya que a la educación musical le conciernen valores relacionados con el hecho de considerar a los estudiantes como seres humanos miembros de una sociedad civil y como

\footnotetext{
${ }^{3}$ Perteneciente o relativo a la teleología: doctrina de las causas finales.

${ }^{4} \mathrm{~N}$. de la A.: Estos principios pueden ser extrapolables a investigaciones realizadas en otras áreas de conocimiento.
} 
ciudadanos que deben ser entrenados para vivir en democracia, y la investigación cualitativa tiene concomitancias con los valores de la comunidad que estudia. Asumir la existencia de múltiples realidades, según Aróstegui (2004), promueve la democracia y se dirige, no sólo a las escuelas en una sociedad democrática, sino también a la música, a la educación y a la investigación.

\section{EL ESTUDIO DE CASOS}

El estudio de casos es una estrategia de indagación (Denzin \& Lincoln, 1994) que utiliza fundamentalmente la metodología cualitativa. Según Stake (2005), se trata de una forma de hacer investigación que busca el estudio de la particularidad y de la complejidad de un caso singular. Se estudia un caso cuando tiene un interés en sí mismo y nos preocupa lo suficiente como para que lo convirtamos en objeto de estudio. El estudio de casos se considera especialmente apropiado para realizar investigaciones en ciencias sociales.

En educación los casos de interés suelen ser personas y programas. Un caso puede ser un niño, un grupo de alumnos, un profesor, una escuela o un programa innovador.

El caso es algo con un funcionamiento específico y complejo, que se define en base a las siguientes características (Stake, 1998:49-50):

- Es holístico en cuanto que es global, se orienta hacia el caso que tiene que ser definido dentro de un contexto. No tiene por qué ser unipersonal. No es muy analítico, ni elemental, ni muy comparativo. No busca comprender cómo se diferencia de los demás, sino cómo es en sí mismo. Sus contextos son descritos con detalle.

- Es empírico, pues se orienta hacia el campo, pone énfasis en lo observable, se esfuerza por ser natural y en no ser intervencionista. Prefiere la descripción con un lenguaje natural, exento de grandes invenciones conceptuales.

- Es interpretativo, ya que tiene en cuenta diferentes perspectivas y visiones de la realidad. Las cosas no son como parecen. Se buscan perspectivas complementarias. Se parte de que el conocimiento es una construcción humana ya que siempre interpretamos la realidad. Los investigadores deben permanecer alerta para reconocer los sucesos que puedan ser importantes. Reconocen que la investigación es una "interacción" entre el investigador y lo investigado, aunque deben preservar las realidades múltiples, las diferentes visiones e incluso las contradicciones.

- Es empático, pues no es intervencionista y atiende a la intencionalidad del actor buscando sus percepciones, sus estructuras de referencia, sus valores. A pesar de estar planificado, su diseño es emergente, está pendiente de confirmación, focalizado progresivamente. El informe provee al lector de una experiencia indirecta. 
Stake (1998:16, 2005:237) establece intereses distintos a la hora de elegir un caso. Puede que en ese momento no tengamos elección y que el caso venga dado, no porque con su estudio aprendamos algo sobre otros casos o sobre un problema en general, sino porque necesitamos aprender sobre ese caso en particular. La elección se haría a partir del interés intrínseco por el caso. Así, nuestro trabajo sería un "estudio intrínseco de casos". Otras veces, la necesidad del estudio vendrá porque frente a una cuestión, situación, o interés por comprender algo, consideramos que podemos hacerlo mediante el estudio de un caso particular. La investigación sería un "estudio instrumental de casos". Puede que en esta misma situación nos parezca adecuado elegir varios casos para estudiar lo que queremos, y entonces se trataría de "un estudio colectivo de casos". Esta distinción nos será útil con respecto a los métodos que vamos a emplear, que serán diferentes dependiendo de si nuestro interés es intrínseco o instrumental.

No es probable que la muestra de un caso, o de unos pocos, sea representativa de otros, porque la investigación con estudio de casos no es una investigación de muestras en el sentido de representatividad numérica de la estadística inferencial. Según Eisner (1998:230), el estudio de casos utiliza el principio de la "transferencia" entendida como un proceso que sirve para reconocer la similitud entre una situación y la siguiente, por medio del cual el investigador no determina la generalizabilidad, sino que es el lector quien decide si las conclusiones o interpretaciones del caso son transferibles a su propio contexto.

En los estudios de caso cualitativos el investigador aprende de lo que éstos tienen que enseñar, pero será el lector quien determine si los hallazgos de la investigación coinciden con la situación que se ha presentado.

\section{LAS TÉCNICAS DE RECOGIDA DE DATOS}

Las técnicas de recogida de datos asociadas con la metodología cualitativa proporcionan información de situaciones y contextos concretos, permiten una descripción exhaustiva y densa -utilizando la terminología de Geertz (2001)- de la realidad objeto de estudio y, a través del texto, proporcionan al lector una "experiencia vicaria" de haber estado ahí (Bresler, 2002:46). Esto requiere, por parte del investigador cualitativo, desarrollar la pericia suficiente para llevar a cabo un empeño muy complejo, distinto de un simple listado de hechos o acontecimientos.

\section{La observación}

La observación es una técnica de recogida de datos que se usa para obtener información sobre individuos o ambientes. La observación resulta muy útil en investigación educativa para analizar, por ejemplo, la interacción en el aula, las estrategias didácticas o los comportamientos docentes. El investigador cualitativo registra minuciosamente los acontecimientos para poder hacer una descripción exhaustiva que posibilite los futuros análisis y el informe final y se sirve para ello de determinados recursos tecnológicos. Como el registro anecdótico -que descri- 
be los acontecimientos relevantes para la investigación- o las grabaciones de audio y vídeo.

Hay diferentes tipos de observación, relacionados con los distintos papeles que adopte el investigador. Puede ser directa o indirecta según se tome contacto personalmente con el objeto de la investigación o a través de observaciones previas realizadas por otros; participante o no participante según interactúe con los sujetos para obtener información desde dentro o se mantenga al margen. Puede ser estructurada o no estructurada según se atenga o no a un proyecto preciso y a una tipología de informaciones a recoger. Puede ser de campo o de laboratorio según se sitúe en el lugar donde ocurren los hechos o fenómenos investigados o en lugares preestablecidos.

\section{La entrevista}

La entrevista es una técnica de recogida de datos que se realiza con aquellas personas que colaboran en la investigación con el fin de recolectar datos que la observación no puede proporcionar para que sirvan como complemento mutuo. La entrevista como técnica de recogida de datos ha despertado un interés singular por parte de la literatura especializada. Existen guías y manuales que contemplan las distintas tipologías de entrevista, las estrategias para realizar una buena entrevista o la manera de discernir qué entrevista es más adecuada para nuestras necesidades y propósitos en cada momento concreto de la investigación.

La tipología de las entrevistas varía en función del enfoque y de la planificación. Una entrevista puede ser individual o grupal; estructurada, semiestructurada o abierta; planificada o espontánea; formal o informal; y puede realizarse más someramente $\mathrm{o}$ en profundidad.

Para la realización de las entrevistas se pueden utilizar registros tecnológicos como magnetófonos o cámaras de vídeo. El uso de dichos registros puede resultar muy adecuado en el ámbito de la investigación educativa ya que recogen acciones y situaciones difíciles de registrar por escrito. Además, a la hora de transcribir se necesita la escucha o la visualización repetida de determinados pasajes, lo que sólo puede hacerse gracias a un soporte de audio o de vídeo.

\section{Otras técnicas}

Hay otras técnicas de recogida de datos como las notas y los diarios de campo, que son la forma narrativo-descriptiva más común para registrar información diversa en diferentes contextos de investigación. Pueden producirse bajo la forma de anotaciones variadas, no sistemáticas, recogidas a vuelapluma fuera del contexto natural donde se realizan las observaciones sistemáticas. Pueden tomar la forma de diario de campo concebido como un registro continuo y sistemático de eventos, fechas, personas, ideas, anécdotas, o como un relato autoevaluativo y reflexivo.

Casi todos los estudios demandan, también, de una manera o de otra, el análisis de documentos de la más variada procedencia tales como artículos, informes, actas, horarios, programaciones, tableros de anuncios, exposiciones, murales o 
cualquier tipo de registro escrito que pueda surgir por iniciativa personal o como sugerencia del investigador.

\section{EL ANÁLISIS Y LA INTERPRETACIÓN DE LOS DATOS}

Una vez obtenidos los datos, el investigador habrá de proceder a la preparación de los mismos para que puedan ser analizados. El investigador cuenta con la oportunidad de poder transferir los datos a un programa informático procesador de textos tanto si dichos datos se han obtenido en forma de manuscrito -como se produce en el caso de las observaciones, notas o diarios-, como si se han obtenido por medio de un audio o de un vídeo como es el caso de la entrevista, en cuyo caso necesitan, además, ser transcritos.

Cuando los datos han tomado forma de texto se produce un salto de calidad importante y el texto corriente adquiere la entidad suficiente como para ser interpretado. Como señala Eisner (1998), describir e interpretar no es lo mismo, aunque conviene tener presente que toda descripción es interpretativa en sí misma por el hecho de que cuando se registra y se describe ya se está interpretando.

Interpretar lo que se ve entraña gran complejidad. Un mismo gesto puede tener diferentes significados: no es lo mismo un guiño que un parpadeo pues el uno tiene mensaje y el otro no y de lo que se trata es de "saber si la descripción distingue los guiños de los tics y los guiños verdaderos de los guiños fingidos" (Geertz, 2001:29). La descripción etnográfica para Geertz (2001:32), es interpretativa en cuanto que interpreta el flujo del discurso social y lo hace rescatando de sus ocasiones perecederas "lo dicho" en ese discurso para fijarlo en términos que permitan su consulta.

La complejidad es algo inherente al propio proceso de interpretación. Esa complejidad se manifiesta, según Eisner (1998), en que la interpretación de lo que ocurre es rara vez el resultado de acontecimientos únicos -tal es el caso de las aulas y las escuelas- $\mathrm{y}$, además, en que los significados que damos a la acción y los motivos que inferimos son interactivos, y es ahí donde aparece otro componente a considerar: la subjetividad, que responde a un presupuesto que subyace en el paradigma interpretativo en función de las "relaciones del investigador con lo investigado" (Bresler, 2006b:83).

Es necesario asumir, identificar, seguir el rastro y domesticar la propia subjetividad, y para ello se ha de contemplar la relación entre ésta y algunas de las decisiones que se van incorporando en el proceso de interpretación. Esas decisiones, según Peshkin (2000), afectarán a los viejos datos, a la búsqueda de datos nuevos y a los debates entre diferentes razonamientos cuando trate de construir el texto emergente a partir del texto original. Lo que excluya o enfatice tendrá consecuencias en el proceso de interpretación. Así pues, el proceso de interpretación nunca es trivial y siempre implica alternativas, y al asumir y juzgar el transcurso del proceso interpretativo desde la cercanía y la honestidad, el trabajo del 
investigador desarrolla una conciencia reflexiva que contribuye a mejorar los actos interpretativos.

En la investigación cualitativa el investigador, en la búsqueda de precisión y de explicaciones alternativas, necesita disciplina y estrategias que no dependan de la simple intuición y de las "buenas intenciones" (Stake, 1998:94). Estas estrategias se llevan a cabo mediante un procedimiento conocido como triangulación, que sirve para obtener interpretaciones lo más acertadas posibles y garantizar su credibilidad. La triangulación es un proceso mediante el cual el investigador, desde la revisión sistemática, huye de cualquier interpretación superficial para ir a la búsqueda de múltiples y variados significados.

Denzin (1984), identificó diferentes opciones y estrategias de triangulación, como la que se realiza a partir de las diferentes fuentes de datos que consiste en ver si aquello que observamos contiene el mismo significado cuando lo encontramos en otras circunstancias. Se puede recurrir también a otros investigadores para que observen conjuntamente con nosotros, o se les pueden presentar nuestras observaciones para que realicen interpretaciones alternativas. Al elegir coobservadores o revisores con puntos de vista teóricos alternativos, actuamos según lo que Denzin (1989) llamó una triangulación de la teoría. Otra forma de triangulación sería la de técnicas de recogida de datos, que resulta ser la estrategia más aceptada, e incluye fundamentalmente la observación, la entrevista y la revisión de documentos para poder ver aquello que no habíamos percibido o, al menos, no de la misma manera.

Otra forma de otorgar credibilidad al estudio en su conjunto es la búsqueda sistemática de casos negativos.

\section{EL PROCESO DE LA INVESTIGACIÓN: PENSAR Y ACTUAR METODOLÓGICAMENTE}

El trabajo de investigación en el que se sustenta este artículo surge, por un lado, de una necesidad pedagógica derivada de mi práctica docente en la formación del profesorado, dado que muchos de mis alumnos, futuros maestros especialistas en educación musical, muestran reticencias ante el canto, carencias en su formación vocal, mal uso de la voz, e incluso, trastornos vocales severos diagnosticados. Por otro lado, responde, también una necesidad teórica ya que no existe suficiente investigación que documente cómo se traducen en la práctica las recomendaciones del currículo en materia vocal.

Una vez acotado el tema en función de esa necesidad pedagógica y teórica, fueron emergiendo las preguntas que guiaron el proceso de la investigación que, finalmente, se articularon en torno a tres grandes bloques con el propósito de averiguar cómo es el modelo vocal que ofrecen los maestros especialistas en educación musical en el aula cuando hablan y cuando cantan, cómo es la didáctica del canto que se práctica en las aulas de educación primaria, y cuáles son las 
ideas de los docentes respecto al rol del canto en la educación musical y en qué medida se corresponden con sus actuaciones.

Sirviéndome de la metodología cualitativa, utilicé como estrategia de indagación, el estudio de $\operatorname{casos}^{5}$, enfoque que, dada la naturaleza de la investigación, entendía apropiado en función de sus señas de identidad como aproximación holística, empírica, interpretativa y empática. Realicé lo que Stake (1998:16-17, 2005:237-238) denomina un "estudio instrumental de casos" cuando establece los distintos intereses a la hora de elegir uno u otro, ya que la elección de los posibles casos surgía frente a una cuestión específica como era el acercamiento a la naturaleza de la práctica vocal que realizaban los maestros en las aulas de educación primaria y respondía al interés por comprenderla. Para este propósito consideré conveniente elegir varios casos, por lo que el trabajo puede considerarse "un estudio colectivo de casos".

\section{Criterios para la elección de los casos}

El trabajo de campo tuvo lugar en centros de educación primaria de la Comunidad de Madrid donde se desarrollaba la actividad docente de maestros especialistas en educación musical. Previo a la elección de los participantes establecí determinados criterios sobre cuáles iban a ser los posibles casos que pudieran servir para la comprensión de aquello que pretendía estudiar siguiendo el principio de máxima variación descrito por Lincoln y Guba (1985) que atiende a la diversidad de los participantes y de los centros donde va a tener lugar la investigación, y tal como preconiza Stake (1998) cuando sugiere la elección de un conjunto de casos equilibrado y variado. Para obrar en consecuencia con el mencionado principio, solicité, en un primer momento, la participación en la investigación de dos maestras especialistas en educación musical que concedían gran relevancia al canto dentro de su práctica, y que poseían perfiles y formación contrastantes.

Comencé con sendos estudios de caso a los que denominé casos piloto no sólo porque fueron mi primera incursión en el campo y en el objeto de estudio, sino también porque, a posteriori, resultaron referenciales dentro del conjunto de casos analizados. Esta primera etapa de investigación fue presentada en un informe para el Examen de Suficiencia Investigadora conducente a la obtención del Diploma de Estudios Avanzados de Doctorado (DEA). Sus conclusiones me permitieron contemplar, en los inicios de la segunda etapa de la investigación, una serie de parámetros para la elección de los posibles casos y para el seguimiento de cada uno de ellos. Dichos parámetros se concretaron en función del principio de máxima variación para ir en busca del contraste, y para ello tuve en cuenta tanto la formación y el perfil vocal, la edad y el género de los participantes, como las características y la ubicación de los centros.

\footnotetext{
${ }^{5}$ Véase el apartado 3: El estudio de casos.
} 
Consideré la oportunidad de buscar perfiles contrastantes desde el punto de vista de la formación académica en general y de la formación vocal en particular, así como prácticas vocales no tan modélicas, si las hubiere, o casos en los que la práctica vocal fuese poco frecuente o inexistente. Así mismo contemplé ampliar las franjas de edad de la muestra ya que, si contaba con la colaboración de maestros más jóvenes tendría más posibilidades de vincular su práctica actual con la formación recibida en su momento y observar con atención a aquellos que se formaron ya como maestros especialistas ${ }^{6}$. También tuve en cuenta el contraste de género a la hora de solicitar la colaboración de maestros varones para poder comprender cómo se establecía su especificidad vocal, en tanto que varones, en relación con la práctica vocal en las aulas de educación primaria.

En cuanto a las características de los centros, consideré también la conveniencia de buscar el contraste y contemplé la oportunidad de realizar algún estudio en centros privados o concertados, e ir en busca de proyectos educativos singulares y acercarme así a su manera de entender el canto. Dado que estos proyectos pueden surgir de planteamientos fundamentados, rigurosos y avalados por su permanencia en el tiempo, tuve en consideración la conveniencia de adentrarme en esos islotes de práctica pedagógica diferente que, supuestamente, podrían aportar modelos de práctica vocal diferentes en relación con los centros públicos. Respecto a la ubicación de los centros, tuve presente abarcar un espectro lo más amplio posible, incluyendo tanto la ciudad de Madrid como otras ciudades de la Comunidad Autónoma. Esta decisión metodológica respondía a una disposición consciente, en tanto que investigadora, con el fin de evitar criterios estratégicos tales como la cercanía, la casualidad o cualquier otra circunstancia coyuntural a la hora de elegir los centros que colaborarían en la investigación.

Desde el acercamiento a las prácticas vocales de los dos casos en la primera etapa de la investigación, emergieron cuestiones relevantes tanto desde el punto de vista teórico como desde el punto de vista metodológico, que resultaron ser cruciales tanto para la elección de los siguientes casos como para su seguimiento. La muestra se fue configurando a lo largo de todo el proceso de investigación en base a los mencionados criterios y principios, y se constituyó, finalmente, en un conjunto de seis casos en los que colaboraron sendos maestros especialistas en educación musical.

En el acercamiento inicial a los maestros me serví de una serie de estrategias fundamentadas en dos cuestiones metodológicas que están relacionadas: garantizar el acceso a una muestra amplia y variada, y crear las condiciones que facilitasen la inmersión en el campo de una manera no casual. Para ello consideré opor-

\footnotetext{
${ }^{6}$ La tesis doctoral se realizó en el periodo en el que estaba vigente la Ley Orgánica de Ordenación del Sistema Educativo (LOGSE) del 3 de octubre de 1990, publicada en el Boletín Oficial del Estado (BOE) el 4 de octubre del mismo año. Esta ley contemplaba en su artículo 16 que "la enseñanza de la música [...] sería impartida por maestros con la especialización correspondiente".
} 
tuno contactar con los maestros personalmente o, si esto no resultaba posible, a través del teléfono, en un intento por evitar utilizar otras modalidades que, entendía, podían resultar más frías e impersonales como, por ejemplo, una carta circular o un correo electrónico. En la búsqueda de estrategias que facilitasen un acercamiento inicial lo más personalizado posible logré, en todos los casos, presentarme siempre de parte de alguien que los maestros conociesen, lo que garantizaba una referencia previa mutua, o propicié el contacto directo anterior al trabajo de campo asistiendo a diferentes cursos concebidos para maestros especialistas en educación musical con los que iba a tener, además, la oportunidad de interactuar en contextos didácticos estimulantes. Esto resultó ser una tarea laboriosa, pero de gran utilidad, a la hora de desempeñar los planteamientos metodológicos respecto a la muestra y al acceso al contexto. Por medio de las estrategias descritas, no sólo obtuve una amplia y variada muestra, sino que, además, esas conversaciones previas, en sus dos modalidades, allanaron el camino y fueron el preludio de la creación de esa zona interpretativa interactiva por la que aspiraba a transitar con los maestros que formarían parte de la investigación, para cuyo acceso, según Bresler (2002:61), en una primera etapa, "resulta decisivo cómo te presentas y cómo llegas".

Los maestros, no sólo ofrecieron su disponibilidad y brindaron todo tipo de facilidades, sino que, incluso, acogieron con agrado la propuesta de participar en la investigación. El acceso al contexto resultó libre de obstáculos y, en el proceso de negociación de dicho acceso, sólo se me pidió expresamente por parte de la dirección de uno de los centros que no utilizase grabaciones de vídeo en las sesiones de observación, algo que, en cualquier caso, no estaba previsto en mi diseño. Por mi parte, asumí por escrito una serie de compromisos previos que salvaguardaban el anonimato, tanto de los colegios como de los maestros. Por eso, en el desarrollo de los informes, evité las referencias a cualquier dato concreto y utilicé seudónimos para referirme a los maestros. Estos compromisos garantizaban tanto mi intención de no interferir en el funcionamiento del centro como la ausencia de cualquier acción unilateral por mi parte sin el consentimiento de los involucrados. Tuve especial cuidado en que los términos del escrito se ajustasen a los criterios de claridad, concisión, compromiso y realismo propuestos por Eisner (1998:204). Cuando el escrito llegaba a la dirección del centro lo hacía de la mano del maestro con el que previamente había establecido contacto, una vez había transcurrido con éxito esa primera fase de acercamiento anterior al trabajo de campo.

Durante todo el proceso de la investigación tuve presente por encima de cualquier otra consideración, incluso de las que se relacionaban con la propia investigación, cuidar la comodidad interpersonal y la confianza mutua que se establecieron con tanto esmero antes del acceso al campo propiamente dicho con el propósito metodológico de conservar dicho acceso, lo que en palabras de Eisner (1998:203-5) resulta tan "delicado" como obtenerlo, pero también por cuestiones éticas frente a los maestros y a los centros que colaboraron, en atención a ellos y con la intención, también, de "dejar el sitio limpio" no sólo para mí -uno sabe 
cuándo realiza la primera observación o entrevista pero desconoce cuándo se realizará la última- sino para evitar que, en un futuro, mi falta de habilidad en la gestión pudiese afectar a las oportunidades de acceso de otros investigadores.

El fin del acceso al campo tuvo lugar en todos y cada uno de los casos en el momento preciso en el que el proceso de la propia investigación lo requería. El hecho de no poder anticipar y concretar cuándo iba a producirse la última observación o entrevista, dado el carácter emergente del proceso de la investigación, no generó ningún problema y tuve la oportunidad de seguir acudiendo al campo cuantas veces lo necesité. Actualmente mantengo contacto profesional con los maestros que colaboraron en la investigación e intercambiamos información sobre temas de interés mutuo.

\section{La recogida de datos}

La organización de la recogida de datos obedeció a un plan que se fundamentaba en las preguntas de la investigación sobre la base de qué era lo que necesitaba saber y por qué, y cuál era la mejor forma de recoger la información. Las técnicas de recogida de datos fueron las asociadas con la metodología cualitativa: la observación, la entrevista, las notas y diarios de campo, y el análisis de documentos y materiales.

Utilicé la observación como primera técnica de recogida de datos, a fin de obtener descripciones de cada situación. Realicé una observación directa, no participante y no estructurada aunque, en este sentido, suscribo el pensamiento de Kushner (2002) cuando, al referirse a sus observaciones, indica que todas ellas están "estructuradas" por el interés que el propio investigador tiene por la interacción humana, por sus propios valores y por todo lo que eso lleva consigo. Las observaciones se efectuaron en las aulas de música durante las sesiones impartidas por los maestros especialistas en educación musical en todos y cada uno de los seis cursos que constituyen la etapa de educación primaria. También realicé observaciones en las sesiones de trabajo de los coros en dos casos en los que el colegio contaba con un coro escolar, así como cuando en el centro se estaba realizando un proyecto determinado o cuando, por ejemplo, debido a la existencia en el centro de otro maestro especialista, el desarrollo de la investigación lo requería.

Recogí los datos de forma sistemática, minuciosa y exhaustiva mediante el registro anecdótico para evitar la posible focalización de las observaciones.

Los intervalos entre las observaciones dejaron espacio para realizar las transcripciones adecuadamente, tan pronto como era posible, para no distanciarlas de la sesión en la que había observado y para retomar fuerzas para la próxima recogida de datos.

Utilicé la entrevista como segunda técnica de recogida de datos, en sus variantes abierta y semiestructurada, planificada y espontánea, y formal e informal. Como recurso tecnológico me serví, básicamente, de una grabadora digital cuyo contenido podía ser transferido al ordenador, facilitando de esta forma la transcripción de las grabaciones como datos textuales. En las ocasiones en las que se 
trataba de una entrevista espontánea o informal recurrí al registro escrito inmediato. Realicé las transcripciones tan pronto como me fue posible para no distanciarlas de la sesión en la que había tenido lugar la entrevista, estableciendo como criterio principal que entre las sucesivas sesiones hubiese un intervalo de tiempo suficiente que me permitiese analizarlas adecuadamente. Realicé la transcripción completa de las entrevistas para dar opción, en un futuro, a que pudieran emerger ideas con posterioridad a las primeras inmersiones en los datos.

Para evitar toda formalidad o rigidez, realicé muchas entrevistas y charlas informales en lugares más "cotidianos" (Eisner, 1998:214), como un vestíbulo, de camino hacia cualquier dependencia del centro, en el comedor o en el patio de recreo. Evité plantear directamente la pregunta central de la investigación como sugiere Kushner (2002), eludí los "por qué" e intenté colocarme en un segundo plano como propone Bresler (2002:60). Todos los cuidados a la hora de entrevistar tuvieron su recompensa pues, en la mayoría de los casos, los escenarios de las entrevistas sirvieron para crear esa situación idónea para la expresión que, según Bresler (2002:61), con el tiempo propicia "un estilo propio de investigación" con un lenguaje corporal y verbal que va creando "zonas interpretativas".

Con la utilización de distintas formas de plantear la entrevista -que no llevaban a respuestas simples ni a responder a una pregunta después de otra- experimenté, como investigadora, el valor de los relatos construidos por los maestros. Estos relatos aportaron información abundante que resultó ser variada, relevante y sutil para los propósitos de la investigación y sirvieron, también para dar voz a los maestros. En los registros de las notas y diarios de campo hallé recurrentes anotaciones respecto a la manera espontánea y prolífica en que los maestros vertían esos relatos. Muchas veces me sorprendían con su discurso sin ser requeridos y respondían incluso a las preguntas centrales de la investigación sin que yo las formulase, sin darme el tiempo siquiera de poner en funcionamiento el resorte mágico del cuéntame sobre o qué era aquello de...

En tercer lugar, las notas y diarios de campo resultaron de gran utilidad para mi trabajo aportando detalles de gran sutileza, sirviéndome como herramientas para la reflexión y para la triangulación. Por último, analicé documentos tales como artículos, informes, actas, textos oficiales, horarios, vídeos, grabaciones, programaciones, tableros de anuncios, exposiciones y murales.

\section{El proceso de análisis e interpretación}

El trabajo de campo tuvo lugar durante los años académicos 2006-2007, 20072008, y 2008-2009. Una vez realizada la transcripción de los datos, el corpus documental quedó constituido por una media de 11 documentos por cada caso que sumaron un total de 68 documentos primarios. El proceso de análisis e interpretación de los datos se realizó de forma cíclica y recurrente, y resultó complejo y laborioso dada su naturaleza y su carácter emergente (Peshkin, 2000).

Cuando se trabaja con textos, la primera fase del análisis consiste en identificar segmentos significativos para la investigación a los que se les asigna un código en base a unos criterios temáticos (Saldaña, 2009). Codificar, como acto 
interpretativo, es un acto recurrente, por eso, es preciso volver una y otra vez sobre el código pues la primera vez que identificamos ese segmento significativo en el texto casi nunca le asignamos el código adecuado.

En el proceso de análisis e interpretación de los datos me serví de algunos elementos del estilo de análisis de la teoría fundamentada (GT) propuesta por Glaser y Strauss (1967), utilizándolos desde la perspectiva de la investigación cualitativa y del paradigma interpretativo. Recurrí a las dos estrategias básicas de análisis de dicha teoría como son la realización de comparaciones constantes y el muestreo teórico.

El método comparativo constante es una aproximación mediante la que el investigador codifica y analiza, simultáneamente, los datos con el propósito de desarrollar ideas teóricas. Este método fue diseñado para ayudar al investigador a generar teoría de manera que ésta sea integrada, consistente, plausible, cercana a los datos y en relación íntima con ellos. Después de haber codificado varias veces se registran esas nociones teóricas en un memorándum o "memo" que sirve para recoger las ideas que van surgiendo y para ayudar a clarificar los posibles conflictos en torno a dichas ideas, y se vuelve a codificar. Según Glaser y Strauss (1967), el carácter recurrente de esta forma de investigación implica un replanteamiento constante que no permite seguir una rutina precisa en la tarea conjunta de codificación y de análisis. La estrategia del muestreo teórico consiste en que una vez que el investigador selecciona, codifica y analiza sus datos en función de la teoría emergente, decide qué información elegir a continuación y dónde encontrarla para poder seguir desarrollando esa teoría tal como surge de los datos.

Durante la primera etapa de la investigación los criterios de recolección de los datos vinieron desde la perspectiva del tema en función de mi propio criterio teórico al respecto, mientras que el resto del proceso discurrió a partir de la teoría emergente.

Procuré contar con una lectura previa lo más amplia posible que me ayudase a poder encontrar modelos que me sirviesen para hacer que los datos tuviesen sentido pero evité, en un primer momento, que el marco teórico estuviese demasiado cerca del tema que estaba investigando para que no condicionase el proceso. En el proceso de análisis e interpretación de los datos recurrí al programa informático Atlas.ti, que está especialmente diseñado teniendo en cuenta el procedimiento de análisis datos en la teoría fundamentada. Este programa resultó ser de gran utilidad a la hora de realizar la codificación. Me serví de los planteamientos de Saldaña (2009), al asignar los códigos en función de criterios temáticos, y también en la utilización que hice de las categorías concebidas como una manera de agrupar códigos similares por familias. Así pues, utilicé los códigos y las categorías como dos elementos diferentes del análisis, tarea que el programa me permitía realizar en estos mismos términos, facilitándome, igualmente, esa recodificación sistemática y periódica que posibilitaba que, a medida que codificaba y recodificaba, tanto los códigos como las categorías se fuesen volviendo "más refinados" (Saldaña, 2009:10). 
El programa Atlas.ti me resultó de gran utilidad no sólo para la codificación, sino también para el resto del proceso del análisis de los datos, ya que su diseño estimula al investigador a ir más allá del trabajo de fragmentación y codificación de los datos y anima al analista a construir relaciones sistemáticas entre las categorías de códigos, y entre estos códigos y otros elementos, tales como los fragmentos de datos originales o los memos. El programa informático me proporcionó una gran ventaja a la hora de realizar tareas de manejo, recuperación y análisis de los datos $\mathrm{y}$, también, en tanto que "mecanismo heurístico", para explorar las relaciones entre familias y conceptos y entre conceptos emergentes entre sí, ya que dichas familias podían visualizarse y modificarse (Coffey \& Atkinson, 2005:30-31). Así, las familias que habían ido surgiendo, y que se habían reformulado constantemente desde los datos, se ajustaban a dichos datos y favorecían que la teoría emergente resultase útil, tanto para el avance teórico como para la aplicación práctica.

No consideré el análisis de los textos de los relatos vertidos por los maestros con relación a las estructuras formales, sus propiedades narrativas o la tipología de los relatos porque rebasaba los propósitos de la investigación, pero sí tuve en cuenta, aunque no de manera sistemática, una serie de aspectos tales como el desempeño oral de los narradores y lo que sobre el ambiente cultural y social en el que se desenvolvían revelaba, las habilidades comunicativas verbales y no verbales que tenían, las habilidades expresivas o la manera en la que los narradores construían las representaciones sobre sí mismos, y todo ello se imbricó en el contexto de mis interpretaciones.

\section{Estrategias de triangulación}

De entre las diferentes opciones que existen utilicé fundamentalmente la triangulación de técnicas de recogida de datos, estrategia que incluye básicamente la observación, la entrevista y la revisión de documentos. No obstante, utilicé otras modalidades de triangulación ${ }^{7}$ cuando lo consideré conveniente para el desarrollo del proceso de la investigación. En el caso 2 utilicé la triangulación de informantes cuando realicé una sesión de observación no participante y una entrevista semiestructurada con otra maestra especialista en educación musical que enseñaba en el centro, $y$, también, mediante una observación no participante durante una conversación entre la maestra que colaboraba en la investigación y otro maestro especialista en Lengua Extranjera. En el caso 5 utilicé la triangulación del investigador cuando recurrí a una profesora visitante, procedente de Noruega, como coobservadora. Esta profesora se ofreció a acudir a algunas sesiones, aceptó que analizásemos juntas nuestras respectivas observaciones, y no tuvo inconveniente en permitirme que la entrevistase. Realizamos, además, una triangulación de la teoría pues pudimos contrastar las observaciones efectuadas por ambas en el

\footnotetext{
${ }^{7}$ Véase el apartado 5: El análisis y la interpretación de los datos.
} 
mismo escenario, tiempo y hora desde puntos de vista teóricos alternativos. En el caso 6 asistí a diversas charlas informales entre la maestra y otras compañeras con las que compartía alumnos en tanto que profesora de música o inquietudes en su función de tutora de uno de los grupos, por lo que realicé una triangulación de informantes, y, además, una triangulación de las fuentes de datos cuando acudí a observar en un curso de educación infantil en el que enseñaba la maestra. Asimismo, dentro del "Seminario permanente de análisis cualitativo" (SPAC) impulsado por el director de mi tesis y de las investigaciones que en ese momento estábamos realizando un grupo de estudiantes de doctorado, tuve la oportunidad de presentar al grupo algunas de mis observaciones y entrevistas, lo que me resultó de gran utilidad al darme cuenta de cómo los datos eran vistos por otros y por el hecho de poder contrastarlos desde el punto de vista descriptivo e interpretativo.

\section{Organización temporal}

Dado que la naturaleza del diseño de la investigación es emergente, su devenir temporal no es algo estático ni predecible, de ahí la dificultad de concretar en el tiempo. No obstante se puede reconstruir, a posteriori, la secuenciación de las diferentes tareas y desempeños y su imbricación aun cuando esto represente algunas dificultades ya que, aunque en algunos momentos el trabajo se focaliza más en una u otra dirección, muy frecuentemente se realizan tareas simultáneas, tareas sobre las que siempre hay que volver. A continuación se presenta una síntesis de las etapas fundamentales que configuraron el desarrollo secuenciado de la investigación.

El periodo de preparación tuvo lugar durante el año académico 2005-2006 mediante la asistencia a los cursos del programa de doctorado: "Investigación del aprendizaje y la enseñanza de la música". La asistencia a esos cursos me proporcionó el acceso a la literatura relevante en investigación en educación musical, así como la oportunidad de asistir a seminarios, participar en grupos de trabajo y de discusión, y realizar distintos trabajos bajo la supervisión de los profesores del programa. El tema objeto de estudio de mi investigación para el futuro se mostró ante mí con una claridad meridiana: quería investigar sobre la práctica vocal en educación primaria. Una vez clarificado este extremo tuvieron lugar los primeros movimientos para contactar con algunos maestros especialistas en educación musical.

La primera etapa de la investigación, o etapa de iniciación, tuvo lugar durante el año académico 2006-2007 en el que realicé dos estudios de caso que constituyeron el embrión de la tesis doctoral. Este proceso cristalizó en la redacción del informe final que fue defendido en el Examen de Suficiencia Investigadora.

La segunda etapa de la investigación tuvo lugar durante los cursos 2007-2008 y 2008-2009 y se inició con los planteamientos teóricos, metodológicos y tácticos 
para la ampliación de la muestra ${ }^{8}$. En esta etapa de inmersión en los datos realicé, además, distintos cursos de metodología, de utilización del programa Atlas.ti, y participé en el "Seminario permanente de análisis cualitativo" (SPAC) durante los años académicos 2008-2009 y 2009-2010. Durante el curso 2009-2010 realicé los informes de los cuatro nuevos casos y revisité los dos previos.

La tercera etapa, o etapa de consolidación, consistió en la reelaboración y ampliación del marco teórico específico y del marco epistemológico y metodológico, que permitió volver sobre los casos analizados y realizar el fundido final a la luz del nuevo marco teórico. Esto se realizó durante los cursos 2010-2011 y 20112012. Durante el primer trimestre de 2012 se dio forma a los capítulos, se realizó la imbricación final de los distintos apartados entre sí, se reestructuró todo el trabajo, se ajustaron las cuestiones formales y se escribieron las conclusiones generales. El proceso culminó en abril de 2013 con la defensa de la tesis doctoral.

El proceso de elaboración de un trabajo de investigación tiene un componente iniciático en el que el investigador ha de enfrentarse a diferentes retos. Según Bresler (2002:60), el análisis y la escritura forman parte de un proceso largo, lento y doloroso que va hacia dentro, que requiere de un gran esfuerzo para impulsar la curiosidad y las preguntas, y que "hace transitar al investigador a través de lo desconocido". Esta travesía me ha servido como vía de transformación no sólo como investigadora, sino también como docente y como cantante.

\section{REFERENCIAS BIBLIOGRÁFICAS}

ARÓSTEGUI, J. L. (2004) "Music education as a social phenomenon". En J. L. Aróstegui (Ed.), The social context of music education (pp. 1-10): Champaign, IL: Center for Instructional Research and Curriculum Evaluation.

BRESLER, L. (2002) "The interpretative zone in international qualitative research". En L. Bresler, \& A. Ardichvili (Ed.), Research in international education: Experience, theory, \& practice (pp. 39-82). Nueva York: Peter Lang.

BRESLER, L. (2006a) “ Paradigmas cualitativos en la investigación en educación musical”. En M. Díaz (Ed.), Introducción a la investigación en Educación Musical (pp. 60-82). Madrid: Enclave Creativa.

BRESLER, L. (2006b) "Etnografía, fenomenología e investigación-acción en educación musical". En M. Díaz (Ed.), Introducción a la investigación en Educación Musical (pp. 83-99). Madrid: Enclave Creativa.

\footnotetext{
${ }^{8}$ Véase el apartado 6. 1: Criterios para la elección de los casos.
} 
COFFEY, A., \& ATKINSON, P. (2005) Encontrar el sentido a los datos cualitativos: Estrategias complementarias de investigación. Alicante: Publicaciones de la Universidad de Alicante.

DENZIN, N. (1984) The research act. Englewood Cliffs, NJ: Prentice Hall.

DENZIN, N. (1989) Interpretative biography. Newbury Park, CA: Sage.

DENZIN, N. K., \& LINCOLN, Y. S. (1994) Handbook of qualitative research. Thousand Oaks, CA: Sage.

EISNER, E. W. (1998) El ojo ilustrado: Indagación cualitativa y mejora de la práctica educativa. Barcelona: Paidós.

GEERTZ, C. (2001) La interpretación de las culturas. Barcelona: Gedisa.

GLASER, B., \& STRAUSS, A. (1967) The discovery of grounded theory. Strategic for qualitative research. Nueva York: Aldine

GUBA, E. G., \& LINCOLN, Y. S. (1994) "Competing paradigms in qualitative research". En N. K. Denzing, \& Lincoln, Y. S. (Eds.), Handbook of qualitative research (pp. 105-117). Thousand Oaks, CA: Sage.

KUSHNER, S. (2002) Personalizar la evaluación. Madrid: Morata.

LINCOLN, Y. S., \& GUBA, E. G. (1985) Naturalistic inquiry. Beberly Hills, CA: Sage.

PESHKIN, A. (2000) "The nature of interpretation in qualitative research". Educational Researcher, 29(9), 5-9.

SALDAÑA, J. (2009) The coding manual for qualitative researchers. Thousand Oaks, CA: Sage.

STAKE, R. (1998) Investigación con estudio de casos. Madrid: Morata.

STAKE, R. (2005) “Case studies". En N. K. Denzing, \& Y. S. Lincoln (Ed.), Handbook of qualitative research (pp. 236-247). Thousand Oaks, CA: Sage. 